\title{
TRIVIALITY OF A TRACE ON THE SPACE OF COMMUTING TRACE-CLASS SELF-ADJOINT OPERATORS
}

\author{
Sung MYung
}

\begin{abstract}
In the present article, we investigate the possibility of a realvalued map on the space of tuples of commuting trace-class self-adjoint operators, which behaves like the usual trace map on the space of traceclass linear operators. It turns out that such maps are related with continuous group homomorphisms from the Milnor's $K$-group of the real numbers into the additive group of real numbers. Using this connection, it is shown that any such trace map must be trivial, but it is proposed that the target group of a nontrivial trace should be a linearized version of Milnor's $K$-theory as with the case of universal determinant for commuting tuples of matrices rather than just the field of constants.
\end{abstract}

\section{Introduction}

Commuting self-adjoint operators are constantly encountered in various mathematical and physical researches. As an example of physics, let $H$ be a Hilbert space whose elements represent physical states. In quantum mechanics, observables are represented by self-adjoint linear operators on $H$ and they are simultaneously measurable without deviation if the corresponding operators commute.

A trace-class operator on a Hilbert space is a bounded linear operator for which a trace is well-defined ([3]). The space of such operators is important also because it enjoys a duality with the space of bounded linear operators because, for a given bounded linear operator $T$ on $H$, we obtain a continuous linear functional on the space of trace-class operators defined by $A \mapsto \operatorname{Tr}(A T)$. For such a class of operators, the trace map is one of the most important invariants we may think of and so we may ask if it can be generalized to tuples of commuting operators.

Received April 13, 2009; Revised December 8, 2009.

2000 Mathematics Subject Classification. 47B25, 81Q10, $19 \mathrm{D} 45$.

Key words and phrases. traces, commuting operators, Milnor's K-theory.

This work was supported by INHA University Research Grant. 
Let $X_{l}$ be the collection of $l$-tuples of commuting self-adjoint trace-class linear operators on some Hilbert space $H$. Each element of $X_{l}$ can be represented by a symbol $\left(A_{1}, \ldots, A_{l}, H\right)$, where $A_{1}, \ldots, A_{l}$ are commuting selfadjoint trace-class operators on some Hilbert space $H$. It becomes a monoid if we define the addition by the rule $\left(A_{1}, \ldots, A_{l}, H_{1}\right)+\left(B_{1}, \ldots, B_{l}, H_{2}\right)=$ $\left(A_{1} \oplus B_{1}, \ldots, A_{l} \oplus B_{l}, H_{1} \oplus H_{2}\right)$. There exists a natural projection map from $X_{l}$ to the set of Hilbert spaces and, for each fixed $H$, the set $\left(X_{l}\right)_{H}$ of $l$-tuples of commuting trace-class self-adjoint operators on $H$, i.e., the 'fibre over $H$ ' can be equipped with a structure of a real topological space. $X_{l}$ will be called the space of commuting trace-class self-adjoint operators in the present article.

When $l=1$, we have the usual trace map $\operatorname{Tr}: X_{1} \rightarrow \mathbb{R}$. It is well-known that the trace of a trace-class operator is equal to the sum of its eigenvalues repeated according to their multiplicities $([1])$. We ask whether there exists a 'trace' on $X_{l}$ for $l \geq 2$.

Definition 1.1. A monoid homomorphism $\psi$ from $X_{l}$ into the additive group $\mathbb{R}$ of real numbers is said to be a trace map if the following three conditions are satisfied:

(i) For each fixed Hilbert space $H$, the restriction of $\psi$ on each fibre $\left(X_{l}\right)_{H}$ is continuous.

(ii) $\psi$ is additive, that is, for arbitrary commuting self-adjoint trace-class operators $A_{1}, \ldots, A_{l}, B$ on a Hilbert space $H$, we have

$$
\begin{aligned}
& \psi\left(A_{1}, \ldots, A_{i-1}, A_{i}+B, A_{i+1}, \ldots, A_{l}, H\right) \\
= & \psi\left(A_{1}, \ldots, A_{i-1}, A_{i}, A_{i+1}, \ldots, A_{l}, H\right)+\psi\left(A_{1}, \ldots, A_{i-1}, B, A_{i+1}, \ldots, A_{l}, H\right) .
\end{aligned}
$$

(iii) If $h: H_{1} \rightarrow H_{2}$ is an isomorphism of Hilbert spaces, then

$$
\psi\left(A_{1}, \ldots, A_{l}, H_{1}\right)=\psi\left(h A_{1} h^{-1}, \ldots, h A_{l} h^{-1}, H_{2}\right) .
$$

(iv) If we have commuting $A_{1}(t), \ldots, A_{l}(t) \in G L_{n}(\mathbb{R}[t])$ such that $A_{i}(0)$ and $A_{i}(1)$ are positive-definite symmetric matrices, then we have

$$
\psi\left(\log A_{1}(0), \ldots, \log A_{l}(0), \mathbb{C}^{n}\right)=\psi\left(\log A_{1}(1), \ldots, \log A_{l}(1), \mathbb{C}^{n}\right) .
$$

The reason behind the condition (iv) is that a trace should be realized from a determinant through the exponential function and a determinant of commuting invertible operators of finite rank should be invariant for a polynomial homotopy, i.e., an $l$-tuple of commuting invertible matrices with polynomial entries. The logarithm of a positive-definite symmetric matrix $A$ is given by $S(\log D) S^{-1}$ if $A=S D S^{-1}$ where $D$ is a diagonal matrix with positive real eigenvalues, $S$ is the orthogonal matrix of corresponding eigenvectors and log is the natural extension of the usual real-valued logarithm.

We note that the conditions (iii) and (iv) are automatically satisfied if we require the following stronger condition:

(iii') We have $\psi\left(A_{1}, \ldots, A_{l}, H_{1}\right)=\psi\left(B_{1}, \ldots, B_{l}, H_{2}\right)$ whenever $H_{1} \cong H_{2}$ and $\operatorname{Tr}\left(A_{i}\right)=\operatorname{Tr}\left(B_{i}\right)$ for every $i=1, \ldots, l$. 
If (iii') holds, then since the determinant of $A_{i}(0)$ is equal to the determinant of $A_{i}(1)$ for every $i$, the trace of $\log A_{i}(0)$ is equal to the trace of $\log A_{i}(1)$ for every $i$ and thus we must have $\psi\left(\log A_{1}(0), \ldots, \log A_{l}(0), H_{0}\right)=$ $\psi\left(\log A_{1}(1), \ldots, \log A_{l}(1), H_{1}\right)$.

Under this definition, we will show that any trace map $\psi$ on $X_{l}$ must be identically 0 when $l \geq 2$. The result may seem rather discouraging, but we hope that the use of Milnor's $K$-theory and related tools may be useful in some constructions in the theory of linear operators as discussed in Section 4.

The present article is organized as follows. In Section 2, we briefly define the Milnor's $K$-group $K_{l}^{M}(k)$ and Goodwillie group $G W_{l}(k)$ of a field $k$ and state a theorem that they are isomorphic. Using this result, we show our main theorem that a trace $\psi: X_{l} \rightarrow(\mathbb{R},+)$ must be trivial in Section 3. In the last section, we speculate a possible generalization of the trace map for commuting operators over an arbitrary field and conjecture an existence of a universal group as in the case of the determinant map which was done in [7].

\section{Milnor's K-theory and the Goodwillie groups}

We define the Milnor's $K$-group $K_{l}^{M}(k)$ of a field $k$ as follows ([5]):

Definition 2.1. The $l$-th Milnor's $K$-group $K_{l}^{M}(k)$ is the additive quotient group of the tensor product $\left(k^{\times}\right)^{\otimes l}=k^{\times} \otimes k^{\times} \otimes \cdots \otimes k^{\times}$(l-times) by the subgroup generated by the elements of the form $a_{1} \otimes \cdots \otimes a_{i} \otimes \cdots \otimes a_{j} \otimes \cdots \otimes a_{l} \in$ $\left(k^{\times}\right)^{\otimes l}$ where $a_{i}+a_{j}=1$ for some $1 \leq i<j \leq l$. We denote by $\left\{a_{1}, a_{2}, \ldots, a_{l}\right\}$, called a Milnor symbol, the image of $a_{1} \otimes a_{2} \otimes \cdots \otimes a_{l} \in\left(k^{\times}\right)^{\otimes l}$ in $K_{l}^{M}(k)$.

In particular, $K_{1}^{M}(k)$ is isomorphic to the multiplicative group $k^{\times}$of nonzero elements, but the group operation is written additively, e.g., $\{a\}+\{b\}=\{a b\}$. Also, we have $\left\{a b, c_{2}, \ldots, c_{l}\right\}=\left\{a, c_{2}, \ldots, c_{l}\right\}+\left\{b, c_{2}, \ldots, c_{l}\right\}$ in $K_{l}^{M}(k)$ by the definition.

On the other hand, for a finite dimensional $k$-vector space $V$, let $G L(V)$ be the general linear group which consists of invertible $k$-linear operators on $V$. Then, the Goodwillie group $G W_{l}(k)$ of $k$ is defined as follows $([6])$ :

Definition 2.2. $G W_{l}(k)$ is the abelian group generated by $l$-tuples of commuting operators $\left(A_{1}, \ldots, A_{l}\right)\left(A_{1}, \ldots, A_{l} \in G L(V)\right.$ for various finite dimensional $k$-vector space $V$, subject to the following four kinds of relations.

(i) (Identity Operators) $\left(A_{1}, \ldots, A_{l}\right)=0$ when $A_{i}$ for some $i$ is equal to the identity operator $I \in G L(V)$.

(ii) (Similar Operators) $\left(A_{1}, \ldots, A_{l}\right)=\left(S A_{1} S^{-1}, \ldots, S A_{l} S^{-1}\right)$ for any isomorphism $S: V \stackrel{\sim}{\rightarrow} W$ of $k$-vector spaces.

(iii) (Direct Sum) When $V$ and $W$ are finite dimensional $k$-vector spaces,

$$
\left(A_{1}, \ldots, A_{l}\right)+\left(B_{1}, \ldots, B_{l}\right)=\left(A_{1} \oplus B_{1}, \ldots, A_{l} \oplus B_{l}\right)
$$

for commuting $A_{1}, \ldots, A_{l} \in G L(V)$ and commuting $B_{1}, \ldots, B_{l} \in G L(W)$. 
(iv) (Polynomial Homotopy) $\left(A_{1}(0), \ldots, A_{l}(0)\right)=\left(A_{1}(1), \ldots, A_{l}(1)\right)$ for commuting automorphisms $A_{1}(t), \ldots, A_{l}(t)$ of a free module of finite rank over $k[t]$, where $k[t]$ is the polynomial ring over $k$ with the indeterminate $t$.

In the above definition, note that we may choose only one vector space $V$ from each class of isomorphic vector spaces to resolve any possible set-theoretic problem. A similar remark could have been also applied during the construction of our monoid $X_{l}$. It is shown in [9] that $G W_{l}(k)$ is isomorphic to the motivic cohomology group $H_{\mathcal{M}}^{l}(\operatorname{Spec} k, \mathbb{Z}(l))$. It is one of the most important theorems in algebraic $K$-theory that $K_{l}^{M}(k)$ is isomorphic to $H_{\mathcal{M}}^{l}(\operatorname{Spec} k, \mathbb{Z}(l))([8])$. More precisely, in [6], the following theorem is proved.

Theorem 2.3. The assignment which sends a Milnor symbol $\left\{a_{1}, \ldots, a_{l}\right\}$ to $\left(a_{1}, \ldots, a_{l}\right)$, where $a_{i}$ is considered simply as $1 \times 1$-matrix in each coordinate, gives rise to an isomorphism $K_{l}^{M}(k) \stackrel{\sim}{\rightarrow} G W_{l}(k)$.

For $k=\mathbb{R}$, the field of real numbers, it is known that $K_{l}^{M}(\mathbb{R})$ is isomorphic to $\mathbb{Z} / 2 \oplus U$, where $\mathbb{Z} / 2$ is generated by the Milnor symbol $\{-1, \ldots,-1\}$ and $U$ is a uniquely divisible abelian group ([4]). A Milnor symbol $\left\{a_{1}, \ldots, a_{l}\right\}$ belongs to $U$ if at least one of $a_{1}, \ldots, a_{l}$ is positive. In such a case, the element can be written as $\left\{b_{1}, \ldots, b_{l}\right\}$ where all $b_{1}, \ldots, b_{l}$ are positive. Accordingly, we may write $G W_{l}(\mathbb{R})=U^{\prime} \cup\left(G W_{l}(\mathbb{R})-U^{\prime}\right)$ as a set, where $U^{\prime} \cong U$ is the subgroup of index 2 in $G W_{l}(\mathbb{R})$, which is generated by the $l$-tuples of commuting positivedefinite symmetric operators.

\section{Triviality of a trace map on the space of $l$-tuples of commuting self-adjoint linear operators}

Let $X_{l}$ be the space of $l$-tuples of commuting self-adjoint trace-class linear operators on some Hilbert space $H$ as defined in Section 1.

Theorem 3.1. For $l \geq 2$, there does not exist a nontrivial trace map from the space $X_{l}$ into $\mathbb{R}$ as in Definition 1.1 .

Proof. We define $Y_{l}$ be the submonoid of $X_{l}$ consisting of the symbols $\left(A_{1}, \ldots\right.$, $\left.A_{l}, H\right)$ where $H$ is finite dimensional. Then any trace map $\psi$ on $X_{l}$, when restricted to $Y_{l}$, induces a monoid homomorphism on $Y_{l}$ which, denoted by $\left.\psi\right|_{Y_{l}}$, enjoys the properties (i)-(iv) of Definition 1.1, in case of finite dimensional $H$ 's.

Now let $P_{l}$ be the monoid whose elements are the symbols $\left(A_{1}, \ldots, A_{l}, V\right)$ where $A_{1}, \ldots, A_{l}$ are commuting positive-definite symmetric linear operators on a finite dimensional real vector space $V$, where the addition is given by

$$
\left(A_{1}, \ldots, A_{l}, V_{1}\right)+\left(B_{2}, \ldots, B_{l}, V_{2}\right)=\left(A_{1} \oplus B_{1}, \ldots, A_{l} \oplus B_{l}, V_{1} \oplus V_{2}\right)
$$

whenever it makes sense.

Then there is an obvious monoid homomorphism $P_{l} \rightarrow U^{\prime}$ whose image generates $U^{\prime}$ as a group. In fact, this map is even surjective ([6]). 
We define the map $\log : P_{l} \rightarrow Y_{l}$ by

$$
\log \left(A_{1}, \ldots, A_{l}, V\right)=\left(\log A_{1}, \ldots, \log A_{l}, V_{\mathbb{C}}\right),
$$

where $\log$ is chosen to take values in real symmetric matrices.

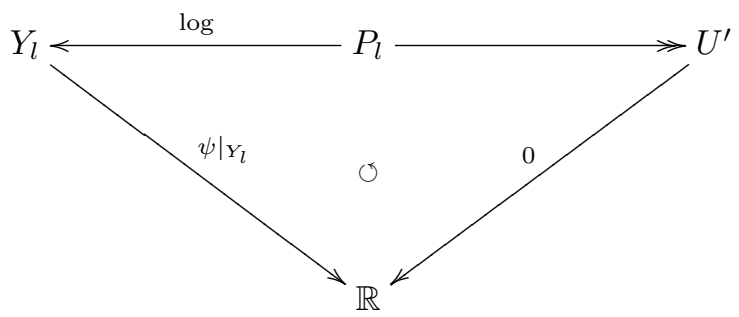

If $\left.\psi\right|_{Y_{l}}: Y_{l} \rightarrow \mathbb{R}$ is the restriction of a given trace map, then the composite $\left.\psi\right|_{Y_{l}} \circ \log : P_{l} \rightarrow \mathbb{R}$ induces a continuous homomorphism from the subgroup $U^{\prime}$ of $G W_{l}(\mathbb{R})$ into the additive group $\mathbb{R}$ of real numbers since the four relations (i)-(iv) in Definition 2.2 are carried to a subset of the relations in Definition 1.1 under the map log: $P_{l} \rightarrow Y_{l}$.

But, Theorem A.1. of [5] shows that any continuous homomorphism from $U^{\prime} \cong U \subset K_{2}^{M}(\mathbb{R})$ into $\mathbb{R}$ must be trivial when $l=2$. The argument there can be easily generalized for $l \geq 3$ and accordingly we conclude that the induced map $U^{\prime} \rightarrow Y_{l}$ is trivial. Thanks to the existence of a simultaneous spectral resolution, for any $\left(A_{1}, \ldots, A_{l}, H\right) \in Y_{l}$, there exists some $\left(B_{1}, \ldots, B_{l}, \mathbb{C}^{n}\right)$ in the image of the log map log $: P_{l} \rightarrow Y_{l}$ such that the operators $\left(A_{1}, \ldots, A_{l}\right)$ and $\left(B_{1}, \ldots, B_{l}\right)$ have the same joint spectrums when counted with multiplicities. Therefore, by Definition 1.1(iii), we conclude that $\left.\psi\right|_{Y_{l}}=0$ on $Y_{l}$.

Now, for each fixed Hilbert space $H$, let $\left(Y_{l}\right)_{H}$ be the subspace of $\left(X_{l}\right)_{H}$ which consists of $l$-tuples self-adjoint operators whose ranges are of finite dimension. By the spectral decomposition, for each $\left(A_{1}, \ldots, A_{l}, H\right) \in\left(Y_{l}\right)_{H}$, there exists a finite dimensional subspace $M$ of $H$ such that $M$ contains all the ranges of $A_{1}, \ldots, A_{l}$ and the operators $A_{1}, \ldots, A_{l}$ if restricted to $M$ are self-adjoint operators. Hence, $\psi$ vanishes on $\left(Y_{l}\right)_{H}$ since $\psi$ is required to be a monoid homomorphism and $\left(A_{1}, \ldots, A_{l}, H\right)$ is a sum of $\left(A_{1}, \ldots, A_{l}, M\right)$ and $(0, \ldots, 0, N)$ where $N$ is a complemental subspace of $M$ in $H$.

Furthermore, by the properties of the trace-class operators as mentioned in [1], $\left(Y_{l}\right)_{H}$ is dense in $\left(X_{l}\right)_{H}$. Consequently, $\psi=0$ on $\left(X_{l}\right)_{H}$ for each $H$ since $\psi$ is required to be continuous on $\left(X_{l}\right)_{H}$ in Definition 1.1(i) and thus $\psi=0$ on the whole $X_{l}$.

\section{Toward a trace as a universal multilinear map}

In this section, we propose a problem of formulating a set of axioms for the trace maps for the commuting operators on finite dimensional vector spaces over an arbitrary field $k$. This generalization would be a general mathematical problem which might extend beyond the interest of a quantum physicist. 
Let us denote by $C_{0 m m}(k)$ be the collection of symbols $\left(A_{1}, \ldots, A_{l}, V\right)$, where $V$ is a finite dimensional vector space over $k$ and is the $l$-tuples of commuting $k$-endomorphisms. We also denote by $\operatorname{Comm}_{l}(k)^{\times}$the subset of $\mathrm{Comm}_{l}(k)$ which consists of commuting automorphisms. Suppose that we have a map $\phi$ from $\operatorname{Comm}_{l}(k)^{\times}$into an abelian group $G$ which satisfies the following four conditions:

(i) For commuting $k$-automorphisms $A_{1}, \ldots, A_{l}, B$ of $V$,

$$
\begin{aligned}
& \phi\left(A_{1}, \ldots, A_{i-1}, A_{i} B, A_{i+1}, \ldots, A_{l}, V\right) \\
= & \phi\left(A_{1}, \ldots, A_{i-1}, A_{i}, A_{i+1}, \ldots, A_{l}, V\right) \cdot \phi\left(A_{1}, \ldots, A_{i-1} B, A_{i+1}, \ldots, A_{l}, V\right) .
\end{aligned}
$$

(ii) $\phi\left(A_{1}, \ldots, A_{l}, V\right)=\phi\left(S A_{1} S^{-1}, \ldots, S A_{l} S^{-1}, W\right)$ for any isomorphism $S$ : $V \stackrel{\sim}{\rightarrow} W$ of $k$-vector spaces.

(iii) For commuting $A_{1}, \ldots, A_{l} \in G L(V)$ and commuting $B_{1}, \ldots, B_{l} \in G L(W)$,

$$
\phi\left(A_{1}, \ldots, A_{l}, V\right) \cdot \phi\left(B_{1}, \ldots, B_{l}, W\right)=\phi\left(A_{1} \oplus B_{1}, \ldots, A_{l} \oplus B_{l}, V \oplus W\right) .
$$

(iv) $\phi\left(A_{1}(0), \ldots, A_{l}(0), M(0)\right)=\phi\left(A_{1}(1), \ldots, A_{l}(1), M(1)\right)$ for commuting automorphisms $A_{1}(t), \ldots, A_{l}(t)$ of a free module $M$ of finite rank over in $k[t]$, where $k[t]$ is the polynomial ring over $k$ with the indeterminate $t$.

Then, Theorem 2.3 and Corollary 2.4 of [6], which states the multilinearity of symbols in the Goodwillie group $G W_{l}(k)$, show that there exists a one-to-one correspondence between the set of maps $\phi: \operatorname{Comm}_{l}(k)^{\times} \rightarrow G$ satisfying the above conditions (i)-(iv) and the set of group homomorphisms from $K_{l}^{M}(k)$ into $G$. For this reason, it is natural to define the 'determinant' as the universal map with respect to the above properties (i)-(iv), i.e., the composite $\operatorname{Comm}_{l}(k)^{\times} \rightarrow$ $G W_{l}(k) \stackrel{\sim}{\rightarrow} K_{l}^{M}(k)$ (See [7] for more details). When $l=1$, it clearly gives the classical determinant.

As for the traces, it is not clear to us what would be a suitable condition which corresponds to (iv) for the determinant. In the important case of the commuting adjoint operators on Hilbert spaces as in the present article, we used an ad hoc definition via the exponential map, or equivalently the logarithmic map. We remark that any trace map for $X_{l}$ in the present article vanished because we insisted that the target of the trace should be $\mathbb{R}$. Hence, we conjecture that there should be a certain universal 'trace' from $C_{0 m} m_{l}(k)$, whose image may be considered as a linearized version of Milnor's $K$-theory, which should play a similar role to the universal determinant $\operatorname{Comm}_{l}(k)^{\times} \rightarrow K_{l}^{M}(k)$. Then our result could have been interpreted as nonexistence of a nontrivial continuous group homomorphism from the 'linearized Milnor's $K$-group' into the field of constants.

\section{References}

[1] J. A. Erdos, On the trace of a trace class operator, Bull. London Math. Soc. 6 (1974), $47-50$. 
[2] R. V. Kadison and J. R. Ringrose, Fundamentals of the theory of operator algebras. Vol. I, Elementary theory. Reprint of the 1983 original. Graduate Studies in Mathematics, 15. American Mathematical Society, Providence, RI, 1997.

[3] _ Fundamentals of the theory of operator algebras. Vol. II, Advanced theory. Corrected reprint of the 1986 original. Graduate Studies in Mathematics, 16. American Mathematical Society, Providence, RI, 1997. pp. i-xxii and 399-1074.

[4] J. Milnor, Algebraic K-theory and quadratic forms, Invent. Math. 9 (1969/1970), 318344.

[5] _ Introduction to Algebraic K-theory, Annals of Mathematics Studies, No. 72. Princeton University Press, Princeton, N.J.; University of Tokyo Press, Tokyo, 1971.

[6] S. Myung, On multilinearity and skew-symmetry of certain symbols in motivic cohomology of fields, Math. Res. Lett. 16 (2009), no. 2, 303-322.

[7] - Transfer maps and nonexistence of joint determinant, Linear Algebra Appl. 431 (2009), no. 9, 1633-1651.

[8] Yu. P. Nesterenko and A. A. Suslin, Homology of the general linear group over a local ring, and Milnor's K-theory, Izv. Akad. Nauk SSSR Ser. Mat. 53 (1989), no. 1, 121-146; translation in Math. USSR-Izv. 34 (1990), no. 1, 121-145.

[9] M. Walker, Motivic complexes and the K-theory of automorphisms, Thesis, University of Illinois, 1996.

Department of Mathematics Education

INHA UNIVERSITY

INCHEON 402-751, KOREA

E-mail address: s-myung1@inha.ac.kr 